\title{
Effect of Lipophilicity of Dispersed Drugs on the Physicochemical and Technological Properties of Solid Lipid Nanoparticles
}

\author{
Annalisa H.S. Stancampiano, Giovanni Puglisi and Rosario Pignatello*
}

Dipartimento di Scienze Farmaceutiche, Università degli Studi di Catania, Catania, Italy

\begin{abstract}
A series of aliphatic esters of the NSAID naproxen (NAP) were synthesised and encapsulated in solid lipid nanoparticles (SLN) to evaluate the influence of lipophilicity on drug loading and release, as well as physicochemical properties and stability of SLN.

The lipophilicity of esters mainly showed to influence their affinity for the solid lipid matrix, modifying the drug release profile compared to the parent drug, whereas drug encapsulation efficiency and the technological properties and stability of SLN were not affected. After storage at room temperature the in vitro release profiles of esters changed with respect to freshly prepared systems, depending on the lipophilicity of the dispersed derivative. In particular, the SLN containing the medium-chain hexyl and octyl esters showed a 70-80\% increase of drug release after storage; SLN loaded with NAP or the lower ethyl and butyl esters did not show significant changes in the drug release rate.

Such findings reveal that the affinity of NAP esters for the lipid network of the nanoparticles is strongly dependent on their lipophilicity and an excessive increase of the latter negatively influences the allocation and retention on these compounds in the nanoparticles.
\end{abstract}

Keywords: Naproxen, aliphatic esters, solid lipid nanoparticles, lipophilicity, stability.

\section{INTRODUCTION}

Solid Lipid Nanoparticles (SLN) have been proposed as an alternative to "traditional" nanoparticulate drug carrier systems, such as emulsions, liposomes and polymeric microand nanoparticles [1].

A clear advantages of SLN is the solid lipid core coated with physiologically compatible and well tolerated emulsifiers. The solid matrix of SLN, essentially based on solid lipids or lipid mixtures, allows to incorporate lipophilic and poorly water soluble ingredients, modulate release profile and avoid their chemical degradation [2].

In this research we tried to systemically evaluate the influence of the lipophilicity of dispersed drugs on their loading in and release from SLN, as well as physicochemical properties and stability of the lipid nanoparticles. One the most recognized limit of SLN, in fact, is the tendency to expulse the incorporated drug molecules during time, as a consequence of a reduction of amorphous regions inside the solid lipid matrix due to polymorphic transitions [3, 4]; this drawback led to the development to alternative systems, like nanostructured lipid carriers or NLC [5].

To perform the present study, we firstly chose as a model drug the non-steroidal antiinflammatory agent naproxen (NAP, Fig. 1). NAP molecule in fact already possesses a lipophilic character $(\log P \sim 3)$ that can allow its incorporation in this kind of carrier. A series of aliphatic esters of

*Address correspondence to this author at the Dipartimento di Scienze Farmaceutiche, Università degli Studi di Catania, Città Universitaria, viale A. Doria 6, I-95125, Catania, Italy; Tel: +39 0957384021; Fax: +39 095222239; E-mail: r.pignatello@unict.it
NAP was then synthesized and incorporated in the SLN. These esters were seen only as model compounds to evaluate the relative effect of lipophilicity on the encapsulation of NAP in the SLN and the following release, and not as potential prodrugs of NAP for therapeutic applications. Thereby, no effort was made to optimize their physicochemical properties, including the very low solubility.

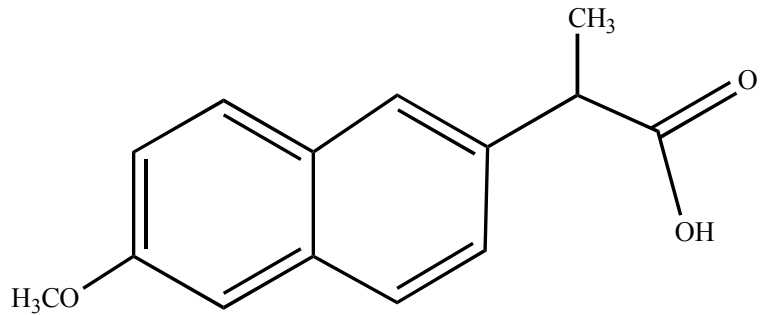

Fig. (1). Chemical structure of naproxen.

SLN were prepared by an opportune modification of the technique of solvent injection [6]. This technique has been already proposed in our laboratory for its simplicity, low quantity of emulsifiers required and possibility of avoiding high temperatures, thus being compatible also with thermosensitive drugs [7].

\section{MATERIALS AND METHODS}

\section{Materials and Methods}

Lipoid S 100 (94\% phosphatidyl choline) was gifted by Lipoid GmbH (Ludwigshafen, Germany). Palmitic acid, NAP and alkyl alcohols were purchased from Sigma-Aldrich Chimica Srl (Milan, Italy). Other solvents and reactants were analytical grade or higher. Solvents were dried by standardized methods [8]. 
Elemental analysis was carried out on a Carlo Erba 1106 analyser; samples were kept in vacuum for $24 \mathrm{~h}$ over $\mathrm{P}_{2} \mathrm{O}_{5}$ before analysis. FT-IR spectra were recorded as nujol mulls with a Perkin-Elmer 1600 spectrophotometer. 'H-NMR spectra were obtained in DMSO- $\mathrm{d}_{6}$ at $200 \mathrm{MHz}$ using a Varian Innova instrument. Chemical shifts are reported in ppm, using tetramethylsilane as internal standard. Purity of compounds was checked by TLC (Merck $\mathrm{F}_{254}$ silica gel aluminium plates) eluted with 1:1 (v/v) dichloromethane-toluene); spots were visualized by UV light.

The partition coefficient and solubility ( $\operatorname{cLogP}$ and cLogS) of NAP and prodrugs were calculated using the $\mathrm{ACD} / \log \mathrm{P} 5.15$ (Advanced Chemistry Development Inc., Toronto, Canada) and the Actelion Property Explorer (www.actelion.com) software packages, respectively (Table 1). The $\mathrm{ACD} / \log P$ database software gives values of $\log P$ in octanol/water, calculated under standard conditions, at $25^{\circ} \mathrm{C}$ and reviews an extensive library of experimental $\log P$ data (www.acdlabs.com). By the Actelion software, the estimated $\log S$ value is a unit stripped logarithm (base 10) of compounds' solubility measured in mol/liter. According to the proprietary indication, the solubility was calculated via an in-house increment system by adding atom contributions depending on their atom types. More than 2000 compounds with experimentally determined solubility values (25 degrees, $\mathrm{pH}=7.5$ ) were used as training set to optimize the contribution values associated with the atom types.

\section{Synthesis of NAP Esters}

NAP esters were prepared according to literature [9]. NAP was first converted into the corresponding acyl chloride by refluxing with thionyl chloride in toluene. NAP chloride was then directly reacted with a 10 -fold excess of the wished alkyl alcohol, for 4 days under stirring at room temperature (r.t.). The resulting white crystals were collected by filtration and recrystallized by 2-propanol. IR and elemental analysis agreed with the literature data [10]. Physicochemical properties of the prepared esters are summarized in Table 1. In the case of the yet undescribed decyl and dodecyl esters, they were synthesised in a similar way and obtained as yellowish oily products:

Decyl 2-(6-methoxynaphthalen-2-yl)propanoate (NAPC10): $\mathrm{C}_{24} \mathrm{H}_{34} \mathrm{O}_{3}$ (370.54) Calcd. C 77.80, H 9.25, Found C 77.78, H 9.27. IR $\left(\mathrm{KBr}, \mathrm{cm}^{-1}\right): 3058 \mathrm{w}, 2962,1733,1630$, 1600; ${ }^{1} \mathrm{H}-\mathrm{NMR}$ (DMSO-d6, ppm): 7.87-7.20 (m, 6H, aromatic), $4.10\left(\mathrm{~m}, 2 \mathrm{H}, \mathrm{OCH}_{2}\right), 3.84\left(\mathrm{~s}, 3 \mathrm{H}, \mathrm{OCH}_{3}\right), 3.78(\mathrm{~m}, 1 \mathrm{H}$, $\left.\mathrm{CH}-\mathrm{CH}_{3}\right), 1.73\left(\mathrm{~d}, 3 \mathrm{H}, \mathrm{CH}-\mathrm{CH}_{3}\right), 1.52-1.26\left(\mathrm{~m}, 16 \mathrm{H}, \mathrm{CH}_{2}\right)$, $0.88\left(\mathrm{t}, 3 \mathrm{H}, \omega-\mathrm{CH}_{3}\right)$.

Dodecyl 2-(6-methoxynaphthalen-2-yl)propionate (NAPC12): $\mathrm{C}_{26} \mathrm{H}_{38} \mathrm{O}_{3}(398.59)$ Calcd. C 78.35, H 9.61, Found $\mathrm{C}$ 78.18, H 9.65; IR $\left(\mathrm{KBr}, \mathrm{cm}^{-1}\right): 3065 \mathrm{w}, 3015,2957,1730$, 1625, 1605; ${ }^{1} \mathrm{H}-\mathrm{NMR}$ (DMSO-d6, ppm): 7.90-7.23 (m, 6H, aromatic), $4.11\left(\mathrm{~m}, 2 \mathrm{H}, \mathrm{OCH}_{2}\right), 3.84\left(\mathrm{~s}, 3 \mathrm{H}, \mathrm{OCH}_{3}\right), 3.80(\mathrm{~m}$, $\left.1 \mathrm{H}, \underline{\mathrm{CH}}-\mathrm{CH}_{3}\right), 1.71\left(\mathrm{~d}, 3 \mathrm{H}, \mathrm{CH}-\mathrm{CH}_{3}\right), 1.50-1.22(\mathrm{~m}, 20 \mathrm{H}$, $\left.\mathrm{CH}_{2}\right), 0.87$ (t, $\left.3 \mathrm{H}, \omega-\mathrm{CH}_{3}\right)$.

\section{Preparation of SLN by the Solvent Injection Method (SI- SLN)}

SI-SLN were prepared by an adapted solvent injection method. Solid lipid (palmitic acid, $20 \mathrm{mg}$ ), the emulsifier (Lipoid S 100, 1\% w/v) and the drug (NAP or its esters, 10 $\mathrm{mg}$, corresponding to a final concentration of $33 \%$, w/v) were dissolved at r.t. in $5 \mathrm{ml}$ of ethanol. The solution was slowly injected by a syringe in $30 \mathrm{ml}$ of bi-distilled water under high speed stirring by an Ultra-Turrax T25 (IKA Labortechnik, Stafen, Germany) at $13500 \mathrm{rpm}$. Solvent residues were evaporated off by slow magnetic stirring for about $24 \mathrm{~h}$ at r.t.

Table 1. Physicochemical properties of NAP Esters

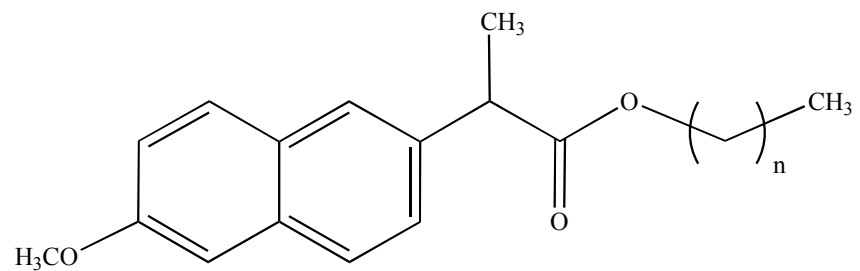

\begin{tabular}{|c|c|c|c|c|}
\hline Compound & $\mathbf{n}$ & MW & $\operatorname{cLog} \mathrm{P}^{\mathrm{a}}$ & $\operatorname{cLog} S^{b}$ \\
\hline NAP & - & 230.27 & 3.00 & -3.59 \\
\hline NAP-C2 & 1 & 258.32 & 3.99 & -4.01 \\
\hline NAP-C4 & 3 & 286.37 & 5.05 & -4.55 \\
\hline NAP-C6 & 5 & 314.43 & 6.12 & -5.09 \\
\hline NAP-C8 & 7 & 342.48 & 7.18 & -5.63 \\
\hline NAP-C10 & 9 & 370.54 & 8.24 & -6.17 \\
\hline NAP-C12 & 11 & 398.59 & 9.30 & -6.71 \\
\hline
\end{tabular}

\section{Characterization of SLN by Photon Correlation Spec- troscopy and $\zeta$-Potential}

The mean particle size of SLN preparations was measured by photon correlation spectroscopy (PCS) with a Zetamaster (Malvern Instruments, UK), equipped with the Malvern PCS software. The dispersions $(200 \mu \mathrm{l})$ were diluted to $3 \mathrm{ml}$ with pro-injection water before the analysis, and the reading was carried out at an angle of $90^{\circ}$. The average diameter and the polydispersity index of SLN were determined and each reported value is the average of thirty measurements.

The electrophoretic mobility was obtained by a laser Doppler anemometer with the same instrument. A suitable amount of each sample $(80 \mu \mathrm{l})$ was diluted with $20 \mathrm{ml}$ of HPLC-grade water and injected in the electrophoretic cell of the instrument. The $\zeta$-potential value was calculated by the instrument software, using Smoluchowsky's equation [11].

\section{DSC Analysis}

Differential scanning calorimetry (DSC) measurements were carried out on a Mettler DSC 12E calorimeter, connected with a Lauda Ecoline RE207 thermocryostat. The detector consisted of a chromel-constantan sensor with a thermometric sensitivity of $56 \mu \mathrm{V} /{ }^{\circ} \mathrm{C}$, a calorimetric sensitivity of about 3 $\mu \mathrm{V} / \mathrm{mW}$, and a noise less than $60 \mathrm{nV}(<20 \mu \mathrm{W})$ at $100^{\circ} \mathrm{C}$. DSC scans showed an accuracy of $\pm 0.4^{\circ} \mathrm{C}$, with a reproducibility and a resolution of $0.1{ }^{\circ} \mathrm{C}$. Indium samples were used to calibrate the instrument. 
Accurately weighed samples of lipid (palmitic acid) and drugs or $40 \mu \mathrm{l}$ of the chosen SLN dispersion were filled into an aluminium pan that was hermetically sealed. The sample was then heated from 20 to $90^{\circ} \mathrm{C}$ at a scan rate of $10^{\circ} \mathrm{C} / \mathrm{min}$. Since the SLN were tested as aqueous suspensions, a highest temperature value below the boiling point of water had to be fixed.

\section{Stability Studies of Esters}

The butyl and decyl esters (about $100 \mathrm{mg}$ ) were dissolved in $1 \mathrm{ml}$ methanol and suspended in $4 \mathrm{ml}$ of HPLC-grade water. The mixture was incubated at $30 \pm 2{ }^{\circ} \mathrm{C}$ under magnetic stirring and, at regular time intervals, $100-\mu 1$ aliquots were withdrawn, diluted with $900 \mu \mathrm{l}$ of methanol, filtered $(0.45 \mu \mathrm{m}$ nylon membrane) and analyzed by HPLC to determine the amount of intact ester and/or produced NAP. A sample of the parent drug was treated similarly for comparison.

In the same study, 1-ml SLN samples loaded with NAP or the two above esters were kept under the same conditions and submitted to HPLC analysis.

A chromatographic procedure previously described was adopted [12].

\section{Drug Encapsulation Efficiency Determination}

A $100 \mu 1$ aliquot of each SLN batch was loaded in a VectaSpin 30K MWCO centrifuge filter (Whatman, Maidstone, UK) and centrifuged at $4^{\circ} \mathrm{C}$ and $10,000 \mathrm{rpm}$ for $60 \mathrm{~min}$. The amount of free and entrapped NAP or esters was determined by dissolving both the liquid phase and pellet with a known amount of ethanol and measuring the absorbance at $272 \mathrm{~nm}$ (Shimadzu UV-1601) vs a calibration curve in ethanol.

\section{Stability Studies of the SLN}

Each SLN preparation was divided in three portions and stored away from direct light at r.t., $4^{\circ} \mathrm{C}$ and $37^{\circ} \mathrm{C}$, respectively. At regular intervals sample aliquots were withdrawn to determine particle size and $\zeta$-potential value.

\section{In Vitro Drug Release}

A dialysis system using Spectra/Por ${ }^{\circledR}$ membrane bags (cutoff: 3,500 Da, Spectrum Laboratories, Inc., USA) was employed. Each bag was filled with $5 \mathrm{ml}$ of SLN suspension and soaked in $50 \mathrm{ml}$ of water/ethanol $(50 / 50, \mathrm{v} / \mathrm{v})$, at r.t. and under a slow magnetic stirring. At regular time intervals, 1-ml aliquots of the external medium were withdrawn and restored with the same volume of water/ethanol mixture. The amount of released drug was measured by UV analysis at $272 \mathrm{~nm}$ (Shimadzu UV-1601).

\section{Statistical Evaluation}

Data are presented as means \pm standard deviation. Statistical analysis between the various experimental results was performed using the analysis of variance (ANOVA). Significance level was set at $\mathrm{p}<0.05$.

\section{RESULTS AND DISCUSSION}

\section{SLN Preparation and Characterisation}

NAP or its esters-loaded SLN were prepared by a modified solvent injection technique and characterized for particle size and homogeneity, z-potential, drug loading capacity and release profile. To ease the comparison among the various batches, the concentration of NAP or the esters in the SLN preparation was kept constant at $33 \%(\mathrm{w} / \mathrm{v})$; such a value come from previous studies of ours (unpublished data), that showed the complete miscibility of NAP with the other ingredients, at this concentration, in analogous systems.

With the aim of better understanding the behaviour of the synthesised esters after their incorporation in the SLN, a preliminary evaluation of their hydrolytic stability was made, to predict any conversion back to the starting NAP molecule under the conditions used for producing the nanoparticles.

NAP butyl and decyl esters, as well as the corresponding SLN, were incubated in water at $30^{\circ} \mathrm{C}$ and analysed by HPLC to measure the extent of hydrolysis. NAP and NAPloaded SLN were used in the same experiments as a control. In these conditions, after 15 days both the esters showed only a limited hydrolysis into NAP (about 6 and 9\% for NAP-C4 and NAP-C10, respectively). In the corresponding SLN, no sign of hydrolytic degradation was observed in the same period. Control NAP did not display any chromatographic change, nor in the free or SLN status. Although the above entities of degradation could be considered unsuitable for a prodrug, it is worthy to note that, within the aim of this study, they can be accepted, in the sense that such a limited hydrolysis would not compromise the interpretation of SLN properties.

The drug loading efficiency was very high for all the esters and similar to the value obtained for NAP (Table 2). No direct relationship between the lipophilicity of esters and their loading in the SLN was evident. Although a reverse behaviour would be expected, with an increase of loading efficacy with increased drug lipophilicity, other properties may affect this parameter, such as surface-related phenomena. Only with the higher homologues the encapsulation efficiency tended to lower, but probably as a consequence of their low solubility in the aqueous medium during the nanoparticle formation, that pushed the partition of these esters in the dispersed lipophilic phase.

Table 2. Drug Encapsulation Efficiency in SLN of NAP or NAP-Esters (Mean of 2-3 Preparations)

\begin{tabular}{|c|c|c|}
\hline $\begin{array}{c}\text { Loaded } \\
\text { Compound }\end{array}$ & $\begin{array}{c}\text { Drug Loading } \\
\pm \text { SD }(\boldsymbol{\mu g} / \mathbf{1 0 0} \boldsymbol{\mu l})\end{array}$ & $\begin{array}{c}\text { Encapsulation } \\
\text { Efficiency (\%) }\end{array}$ \\
\hline \hline NAP & $29.08 \pm 0.11$ & 87.25 \\
\hline NAP-C2 & $23.85 \pm 0.08$ & 71.56 \\
\hline NAP-C4 & $26.95 \pm 0.15$ & 80.86 \\
\hline NAP-C6 & $32.76 \pm 0.23$ & 98.30 \\
\hline NAP-C8 & $32.56 \pm 0.08$ & 97.70 \\
\hline NAP-C10 & $23.91 \pm 0.31$ & 71.70 \\
\hline NAP-C12 & $25.12 \pm 0.11$ & 76.40 \\
\hline
\end{tabular}

Immediately after the preparation, the SLN showed a mean size between 175-195 nm (Fig. 2). In a previous study, we compared the SI technique with a microemulsion process to form SLN and observed that the former allowed to gain a lower particle size [7]. Most probably, the high agitation 
speed in the SI technique, along with the fact that lipids and surfactants are in a solution state instead of melted, could favour a better dispersion of lipid molecules and their following precipitation into smaller particles.

To evaluate the stability of these formulations, SLN were stored either at r.t., at $4{ }^{\circ} \mathrm{C}$ or at $37^{\circ} \mathrm{C}$. After 4 months of storage at r.t. the nanoparticle size showed only a moderate tendency to increase in respect to the freshly prepared SLN (Fig. 2); particle size however did not exceed a value of 250 $\mathrm{nm}$ also after storage.

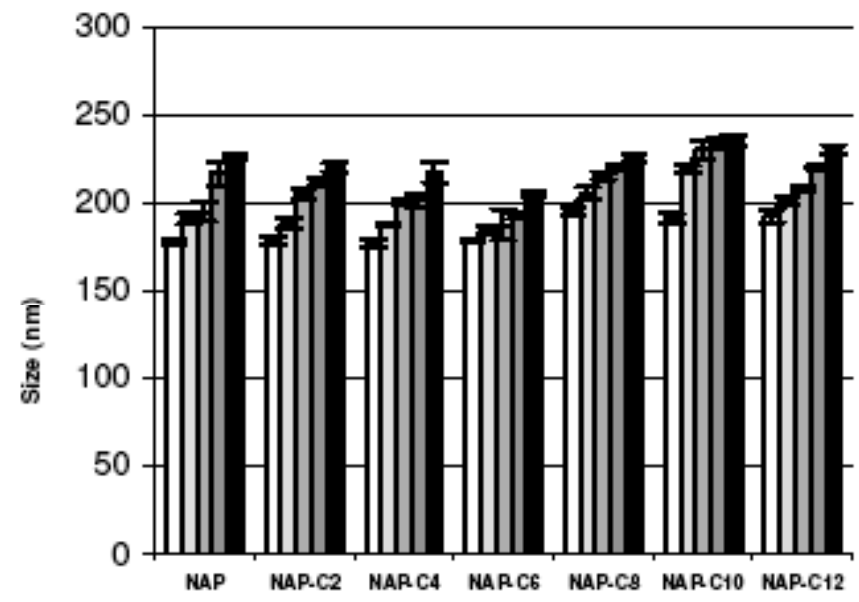

Fig. (2). Mean size changes of SLN during 4 months of storage at r.t.. The bars respectively refer to the values measured after the preparation and then after $1,2,3$, and 4 months.

Also the polydispersity index remained around 0.2 , a value that substantiate a great size homogeneity of these systems (Fig. 3).

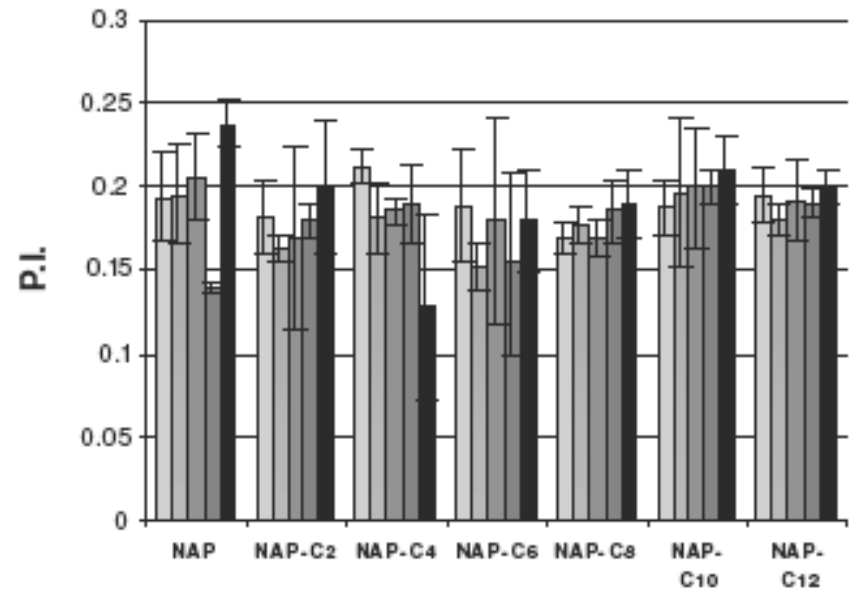

Fig. (3). Polidispersity index of SLN during 4 months of storage at r.t. The bars respectively refer to the values measured after the preparation and then after 1,2,3, and 4 months.

All the drug-loaded SLN showed a negative mean surface charge (around $-25 /-30 \mathrm{mV}$ ), not far from that one measured for plain nanoparticles [7] and easy to interpreter considering the presence of palmitic acid in the SLN matrix composition. Particle surface charge showed only very limited changes upon storage at r.t. (Fig. 4); in particular, the values measured after 4 months were statistically significant $(\mathrm{p}=0.02$, F-test) compared to the initial ones. Only the SLN loaded with NAP displayed an increase of the net negative surface charge during the time. Due to the anionic properties of this carboxylic drug, such a behaviour could indicate a gradual transfer of the dispersed drug toward the surface of the nanoparticles.

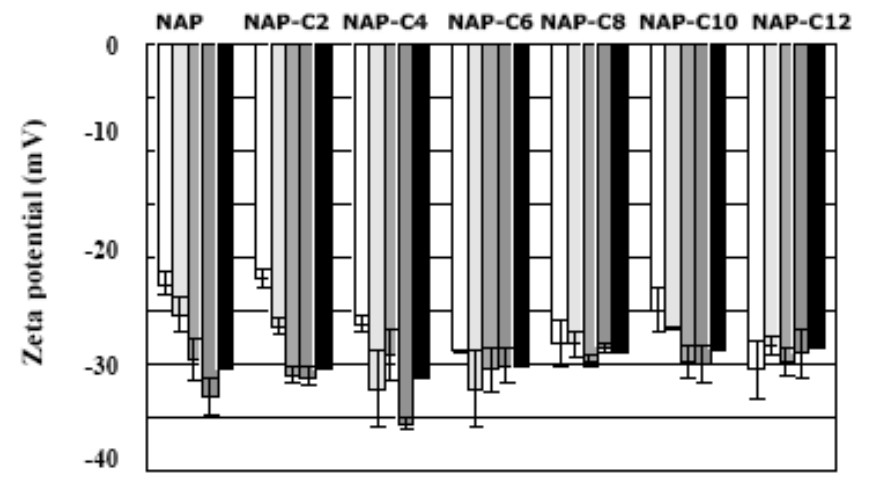

Fig. (4). Zeta potential of SLN during 4 months of storage at r.t.. The bars respectively refer to the values measured after the preparation and then after 1, 2, 3, and 4 months.

Stored at $4{ }^{\circ} \mathrm{C}$ all the colloidal systems underwent a rapid alteration, shown by flocculation and a strong size increase (up to $2 \mu \mathrm{m}$ ); at $37^{\circ} \mathrm{C}$ the SLN were stable for 2 months, after which a sedimentation and size increase was registered, again suggesting an aggregation of nanoparticles.

\section{DSC Studies}

To check the physical status of NAP and its esters within the lipid matrix of SLN, DSC analysis was performed on pure compounds and loaded SLN. As an example, butyl and decyl esters were studies, along with the parent drug.

Thermoanalysis of the bulk lipid (palmitic acid) showed a single fusion endothermic peak centred at $67^{\circ} \mathrm{C}$ (Fig. 5). The DSC curve of plain SLN, as well as of the SLN loaded with NAP did not show any endothermic change. This suggest that all the lipid components are present in the SLN in an amorphous state. On the other hand, also pure NAP did not show any peak within the considered temperature range (Fig. 5), its melting point being much higher $\left(152-153^{\circ} \mathrm{C}\right)$.

An identical consideration could be made for the decyl ester (data not shown), was DSC curves did not gave any signal, neither for the pure compound or the corresponding SLN.

More interesting, the butyl ester of NAP had a clear fusion peak around $62^{\circ} \mathrm{C}$ (Fig. 6). Nevertheless, the incorporation in the SLN suppressed any calorimetric signal, confirming its complete dissolution or amorphisation in the semisolid matrix of the nanoparticles.

\section{In Vitro Drug Release}

The release profile of NAP or its esters from the SLN was assayed immediately after the preparation or after 2 and 4 months of storage at r.t. (Figs. 7 and 8). A dialysis method was used, with an external medium consisting of a 1:1 water/ethanol mixture. All the tested compounds were soluble in such a system, therefore sink conditions were supposed to be maintained during the release experiments.

By considering the reduced solubility in water of the longer-chain esters (Table 1), a strong effect of lipophilicity on their association with the solid lipid matrix, and thus on 
their release in the external aqueous medium was expected. In the freshly prepared SLN the esters showed a reduced drug release, compared to NAP, linearly related to their lipophilicity. Such differences were statistically significant for all the systems $(p<0.05$ for NAP-C2 and NAP-C4 loaded SLN, and $p<0.001$ for the other esters). However, only for the more lipophilic terms the release from SLN was completed in 16 days, whereas shorter-chain homologues still remained partially associated with the lipid matrix, the amount released reaching a maximum of $70 \%$.

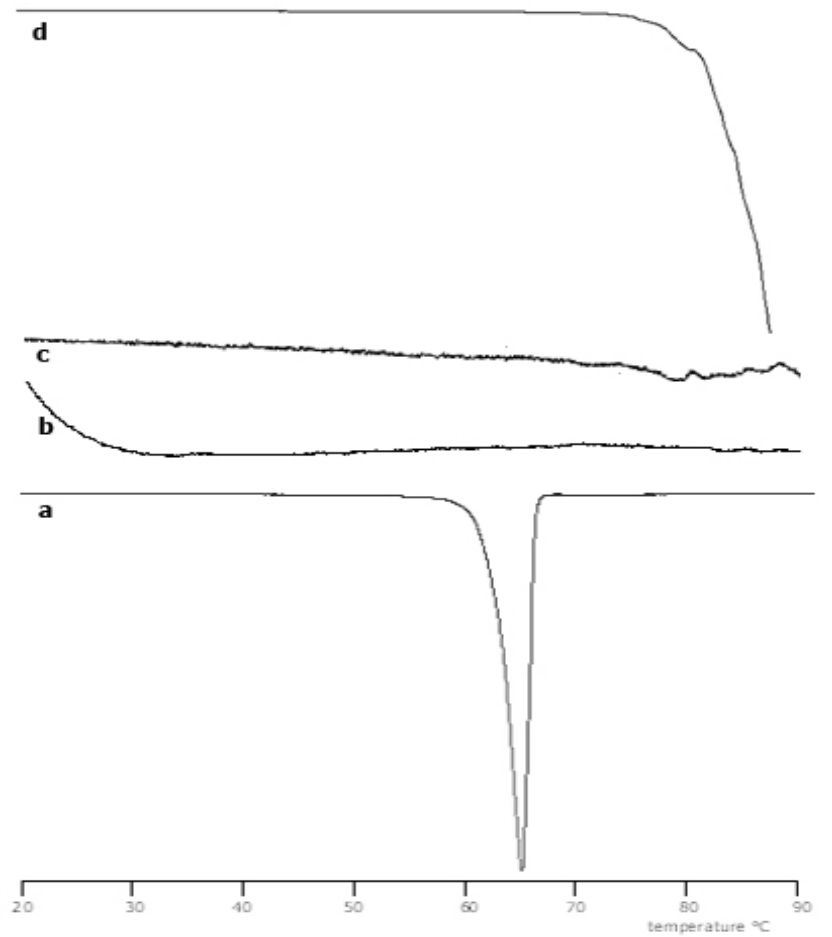

Fig. (5). DSC curves in heating mode (scan rate: $10^{\circ} \mathrm{C} / \mathrm{min}$ ) of (a) palmitic acid, (b) plain SLN made by palmitic acid and Lipoid S $100,(\mathbf{c})$ pure NAP, and (d) NAP-loaded SLN.

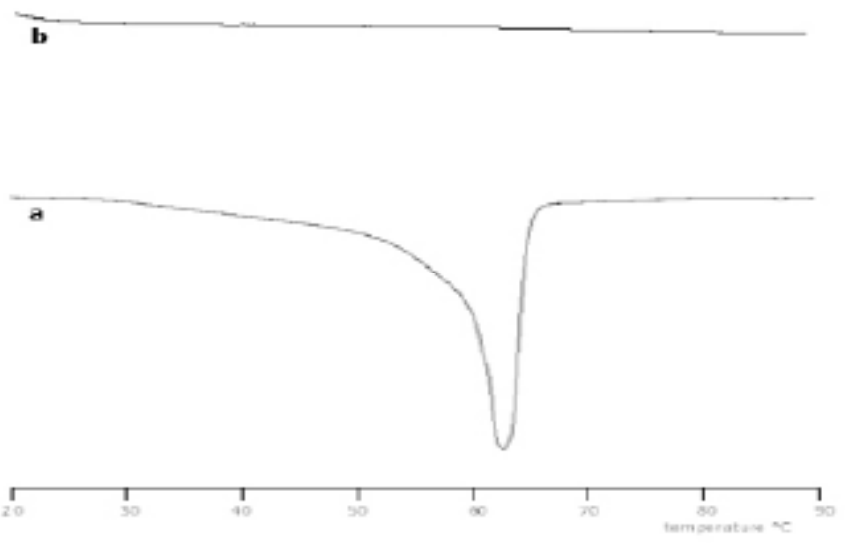

Fig. (6). DSC curves in heating mode (scan rate: $10^{\circ} \mathrm{C} / \mathrm{min}$ ) of (a) NAP butyl ester and (b) SLN loaded with the same compound.

After storage at r.t., the drug release from the nanoparticles was faster and more linear. After 2 months, the higher homologues NAP-C10 and NAP-C12 showed a prompter release from the SLN with respect to the other homologues $(p<0.05)$, which however showed a release profile close to

that of NAP and were completely released within $48 \mathrm{~h}$. ANOVA tests showed that differences were significant for the NAP-C6 and NAP-C8 loaded SLN, while a value of $p>0.05$ was measured for the two lower esters. After 4 months of storage, the release speed was even higher, with a more evident burst phase. For all the systems the experimental data were statistically significant in respect to the initial drug release values. The medium-chain homologues NAPC6, NAP-C8 and NAP-C10 were released completely within $6 \mathrm{~h}$, whereas the other esters showed a complete release in $24 \mathrm{~h}$, always superimposable to the parent drug (Fig. 8).

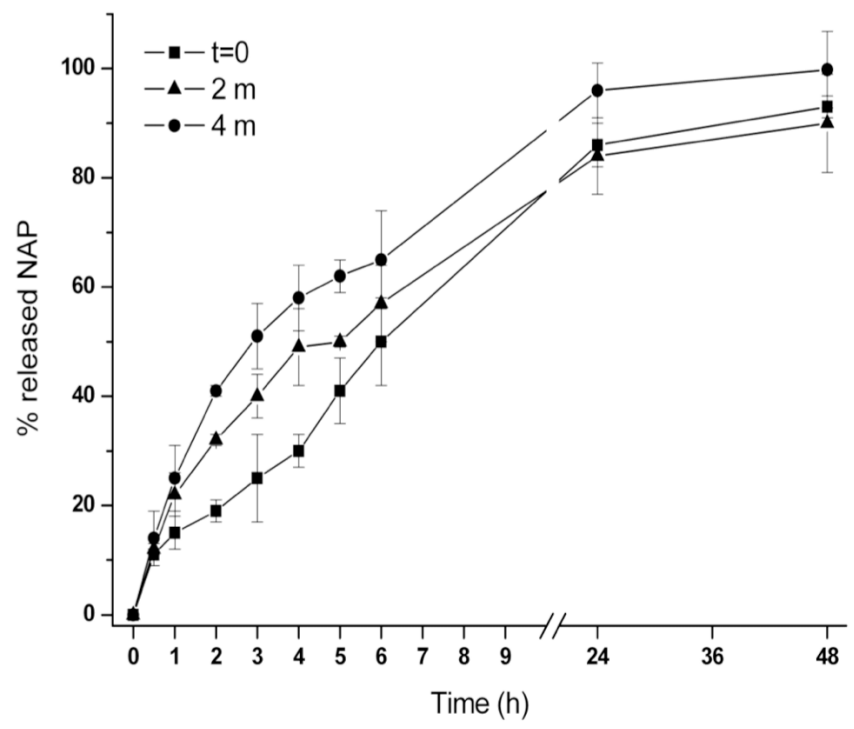

Fig. (7). In vitro release profile of NAP from freshly prepared SLN $(\boldsymbol{\square})$ or after $2(\mathbf{O})$ or 4 months $(\boldsymbol{\Delta})$ of storage at r.t.

To look inside the effect of lipophilicity on ester retention in/release from the SLN, Fig. (9) shows the increase of the percent released esters from nanoparticles stored at r.t. for 4 months, in comparison with the corresponding freshlyprepared systems. Our assumption was than any difference in drug release observed in aged SLN would be the consequence of a partial or limited solubility in or affinity for the solid lipid matrix forming the nanoparticles of the host compounds.

The SLN containing the parent NAP did not show significant changes in the amount of released drug before and after storage $(\mathrm{p}=0.333)$, indicating that the drug is well accommodated in the system and no expulsion phenomenon occurred within the examined period of time.

The ester-loaded SLN gave instead a somehow parabolic profile: the nanoparticles containing the lower-chain ethyl and butyl esters displayed the same behaviour, i.e., a limited increase of drug leakage (20-40\%) from the aged systems, compared to the freshly prepared ones at the same test times. Since the enhancement of the percentage of released drug raised with the test times, these esters should be homogeneously distributed in the lipid matrix and should not have been transported toward their surface; therefore, their leakage was mainly driven by the time of contact of the SLN with the 

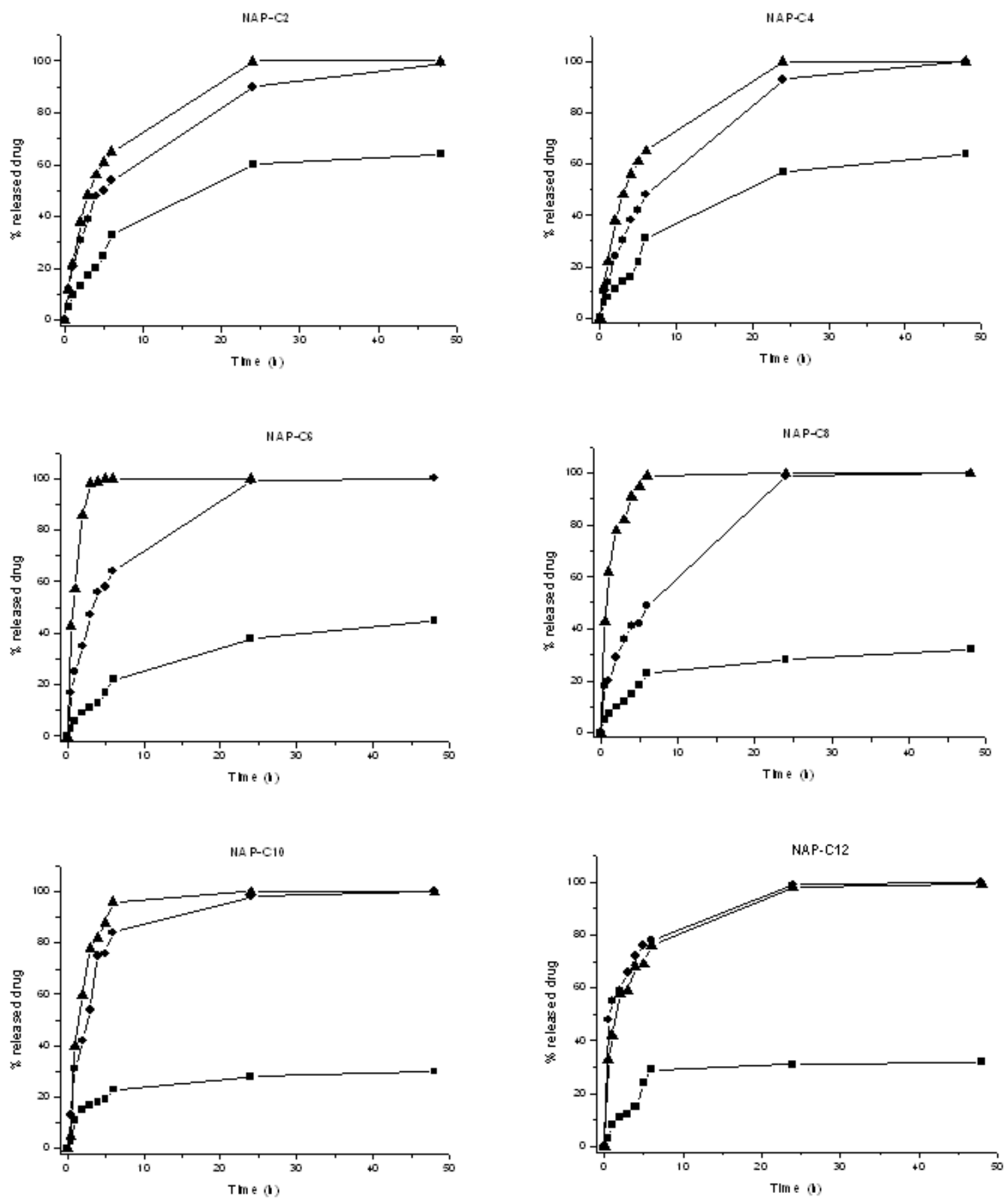

Fig. (8). In vitro release profile of NAP esters from freshly prepared SLN (ם) or after $2(\boldsymbol{O})$ or 4 months ( $(\boldsymbol{\Delta})$ of storage at r.t.

dissolution medium and the consequent diffusion of the esters from the nanoparticle matrix.

Both the SLN loaded with the medium-chain hexyl and octyl esters showed in turn a similar trend, giving a $70-80 \%$ enhancement of drug release after 4 months of storage. Because this was observed as early as at the second hour of test, and was statistically significant $(\mathrm{p}=0.01-0.04)$, it suggests that these compounds, because of their specific physicochemical properties, upon storage were forced towards the surface of nanoparticles, from where they can be released more readily.
Finally, the longest homologues, the decyl and dodecyl esters, displayed a mixed behaviour with respect to the above pairs of esters: in fact, the resulting SLN showed a strong increase of drug release after storage, but the phenomenon was more evident at longer dissolution test times $(6$ and $24 \mathrm{~h}$, $\mathrm{p}<0.05)$ and less after $2 \mathrm{~h}(\mathrm{p}=0.11)$. It is possible that these compounds experienced a double situation: from one side, their nature or structure did not allow a stable allocation within the lipid matrix over time; on the other hand, the high lipophilicity (see Table 1) and thus the low solubility did not facilitate their movements towards nanoparticle surface: as a result, their leakage from the SLN was faster after storage, 
but was also dependent on the time of contact of the nanoparticles with the aqueous dissolution medium.

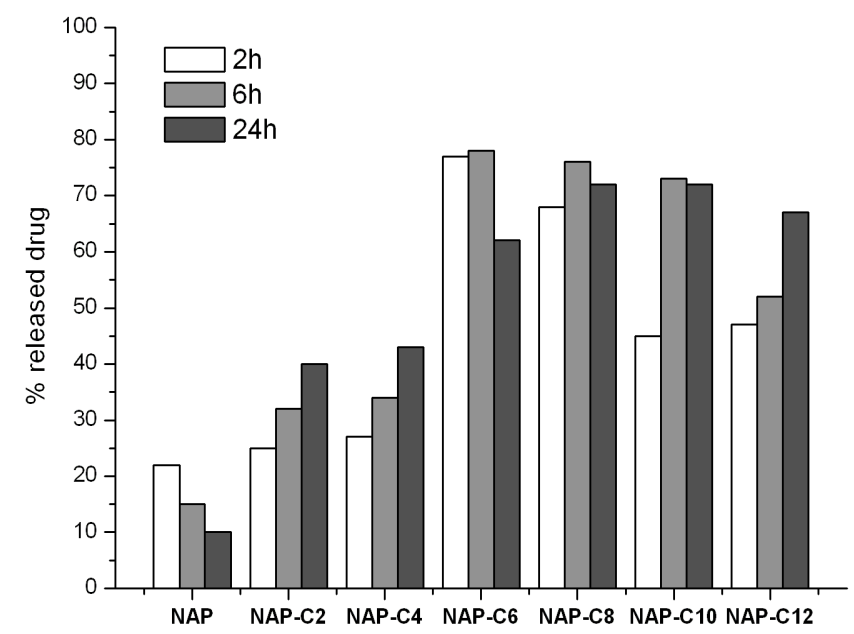

Fig. (9). Effect of storage on NAP or esters release from SLN. Values are reported as the difference in percent release, at different times of the test (2, 6 or $24 \mathrm{~h})$, for the SLN stored for 4 months at r.t., with respect to the amount of each compound released from the corresponding freshly prepared systems.

\section{CONCLUSIONS}

The increased lipophilicity of the synthesized NAP esters affected their affinity for the solid lipid matrix, modifying primarily the drug release profile. Conversely, no significant influence on particle size, surface charge and stability was registered. The esters with a lower lipophilicity were able to remain associated for a longer time with the lipid nanoparti- cles, whereas the highest lipophilic homologues were expulsed more easily from the nanoparticles upon storage.

Within the prepared series of homologues, results suggested that the further increase of lipophilicity in the already lipophilic molecule of NAP did not give advantages either in terms of loading or retention in a lipid carrier like SLN. The phenomenon of drug expulsion, reported also for other drugs $[3,4,13]$ seems to be amplified by the increase of lipophilicity and/or steric hindrance of the drugs that must be allocated inside the lipid matrix. On the other hand, an interesting result observed is that, because of or although their highly lipophilic character, these esters did not induce physical destabilization of the SLN systems in the mid-term storage.

\section{REFERENCES}

Müller, R.H.; Mäder, K.; Gohla, S. Eur. J. Pharm. Biopharm., 2000, 50, 161.

[2] Jenning, V.; Gohla, S. Int. J. Pharm., 2000, 196, 219.

[3] Jenning, V.; Gohla, S. J. Microencapsulation, 2001, 18, 149.

[4] Uner, M. Pharmazie, 2006, 61, 375.

[5] Saupe, A.; Wissing, S.A.; Lenk, A.; Schmidt, C.; Müller, R.H. Biomed. Mater. Eng., 2005, 15, 393.

[6] Pignatello, R.; Bucolo, C.; Spedalieri, G.; Maltese, A.; Puglisi, G. Biomaterials, 2002, 23, 3247.

[7] Stancampiano, A.H.S.; Acquaviva, R.; Campisi, A.; Vanella, L.; Ventura, C.A.; Pignatello, R.; Puglisi, G. J. Biomed. Nanotech., 2006, 2, 253.

[8] Perrin, D. D.; Armarego, W.L.F.; Perrin, D. R. Purification of Laboratory Chemicals, 2nd Ed., Pergamon Press, Oxford, 1980.

[9] Spahn, H. Arch. Pharm., 1988, 321, 847.

[10] Weber, H.; Meyer-Trumpener, K. Arch. Pharm., 1994, 327, 337.

[11] Hunter, R.J. Zeta Potential in Colloid Science; Academic Press: New York, 1981.

[12] Pignatello, R.; Montenegro, L., Stancampiano, A.H.; Puleo, A.; Puglisi, G. Curr. Drug Del., 2005, 2, 185.

[13] Jenning, V.; Schäfer-Korting, M.; Gohla, S. J. Control. Rel., 2000, $66,115$. 